\title{
A Model of one central regulatory circuit
}

\author{
Tatyana Bukharina \\ Institute of Cytology and \\ Genetics SB RAS \\ Novosibirsk, Russia \\ bukharina@bionet.nsc.ru
}

\author{
Andrey Akinshin \\ Sobolev Institute of \\ Mathematics SB RAS \\ Novosibirsk, Russia \\ Andrey.akinshin@gmail.com
}

\author{
Vladimir Golubyatnikov \\ Sobolev Institute of \\ Mathematics SB RAS \\ Novosibirsk, Russia \\ vpgolubyatn@gmail.com
}

\author{
Dagmara Furman \\ Institute of Cytology and \\ Genetics SB RAS \\ Novosibirsk State University \\ Novosibirsk, Russia \\ furman@bionet.nsc.ru
}

\begin{abstract}
Macrochaetes are sensor organs of the drosophila with a function of mechanoreceptors. An adult mechanoreceptor comprises four specialized cells. All these cells originate from the sensory organ precursor (SOP) cell. A characteristic feature of the SOP cell is the highest content of the proneural proteins ASC as compared with the surrounding cells. The accumulation of these proteins and maintenance of their amount in the SOP cell at a necessary level is provided by the gene network with the achaete-scute gene complex $(A S-C)$ as its key component. The central regulatory circuit (CRC) controls the activity of this complex. The CRC activity comprises two phases differing in the time when they act. A specific feature of the second phase is the presence of PHYL protein, involved in degradation of proneural proteins. We propose a mathematical model for the CRC functioning as a regulator of the content of ASC proteins in the sensory organ precursor cell. ${ }^{2}$
\end{abstract}

Keywords - dynamical systems, central regulatory circuit, gene networks models, achaete-scute complex, mutations

\section{Introduction}

The drosophila mechanoreceptors are the result of determined conversion of the ectodermal cells of imaginal discs into progenitor neural cells [1]. The morphogenesis of macrochaetes is a multistage process, where separation of the sensory organ precursor (SOP) cell from ectodermal cells in the wing imaginal disc is the basic event. Production and utilization of the proneural ASC proteins in SOP cell is controlled by a set of genes and the proteins they encode, which are functionally united into $\mathrm{CRC}$. These genes are linked by positive and negative feedbacks mediated via the proteins and protein complexes, activating or repressing transcription of their target genes. In addition to $A S-C$, the CRC contains hairy, senseless (sens), charlatan (chn), scratch (scrt), and phyllopod (phyl) genes. The general scheme of this circuit is shown on the Figure 1 [2]. We propose a mathematical model for the CRC functioning as a regulator of the content of proneural ASC proteins in the sensory organ precursor cell. -

\section{Mathematical Model}

Following the general principles of construction of gene networks models [2-4], we consider the dynamical system for non-negative variables $x_{i}=x_{j}(t)$, here and below $i=1,2, \ldots, 6$ :

It is well known from experimental data that the PHYL protein appears in the cell in 10-12 hours later than ASC proteins $[5,6]$. Thus the CRC functioning can be described by a system of delayed argument $t-\tau$ appears in the first and in the

Supported by RFBR, grant 18-01-00057.

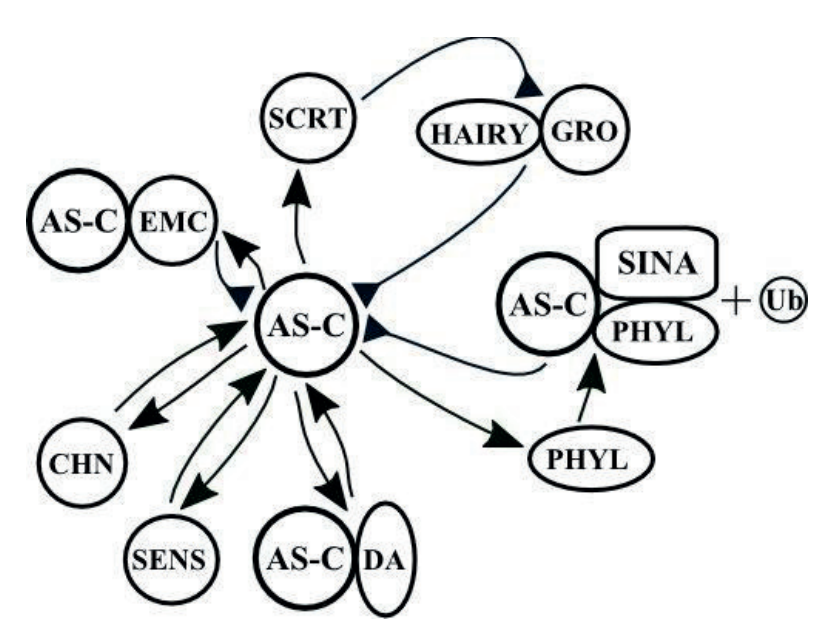

Fig. 1. Central regulatory circuit of the gene system controlling drosophila mechanoreceptors morphogenesis. Arrows denote activation of processes (positive feedbacks), stub lines, repression (negative feedbacks).

last equations on the appropriate places differential equations which is composed on the basis of the scheme depicted on the Figure 1 following general methodology of construction of such models.

$$
\begin{aligned}
& d x_{1} / d t=k_{1} F_{1}\left(x_{1}, x_{2}, x_{3}, x_{5}\right)-m_{1}\left(x_{6}(t-\tau)\right) x_{1} ; \\
& d x_{2} / d t=k_{2} F_{2}\left(x_{4}\right)-m_{2} x_{2} ; \\
& d x_{j} / d t=k_{j} F_{j}\left(x_{1}\right)-m_{j} x_{j} ; j=3,4,5 ; \\
& d x_{6} / d t=k_{6} F_{6}\left(x_{1}, t-\tau\right)-m_{6} x_{6} .
\end{aligned}
$$

CRC proteins content are denoted as follows: AchaeteScute (ASC), $x_{1}(t)$; Hairy, $x_{2}(t)$; Senseless (SENS), $x_{3}(t)$; Scratch (SCRT), $x_{4}(t)$; Charlatan $(\mathrm{CHN}), x_{5}(t)$; Phyllopod (PHYL), $x_{6}(t)$. Content of Ubiquitin (Ub), Seven-in-absentia (SINA), Daughterless (DA), Exstramacrochaete (EMC), and Groucho (Gro) proteins almost do not vary during the considered process, thus we assume that they are constant.

Here, the non-negative coefficients $k_{i}$ reflect status of CRC genes: $k_{i}=1$ when there is no mutation in the $i$-th gene and synthesis rate of corresponding protein is $100 \% ; k_{i}=0$ when the $i$-th gene is mutated and there is no synthesis of corresponding protein at all. Positive functions $F_{i}$ denote velocities of synthesis of CRC proteins. The positive coefficients $m_{i}$ characterize velocities of CRC proteins degradation. Positive summand $k_{2} F_{2}$ in the second equation of the system describes negative feedback between SCRT and HAIRY (Figure 1). 


\section{Software}

In order to fulfill numerical experiments with the model described above, we have elaborated specialized software on the base of the language $\mathrm{R}$ see https://www.r-project.org and the package Shiny (http://shiny.rstudio.com ).

This software is realized as a client -server application:

All calculations are done on the cloud server, and the results are presented at https://andreyakinshin.shinyapps.io/asc_with_delays

Numerical solutions of differential equations with delayed arguments use the package deSolve https://andreyakinshin.shinyapps.io/as-c_with_delays which was already applied to numerical modeling of other gene networks [2].

\section{Numerical Results of Modeling}

Results of numerical experiments with the system for different parameter values of this system are presented at:

https://gist.github.com/AndreyAkinshin/fb2cae36b89838ad 5 dcac706b1d232a1

https://gist.github.com/AndreyAkinshin/a7450a9d234da67f ee8e67ab6f4364f8

https://gist.github.com/AndreyAkinshin/3f2c3c49a954a2c9 $38 \mathrm{c} 5355 \mathrm{c} 1263 \mathrm{f} 554$

Comparison of these results (see links above) shows that:

1. For the coefficient $k_{l}=1$ the graph for content of ASC proteins is adequate to known experimental data. According to them ASC proteins content increases until the appearance of the PHYL protein in the SOP cell and then quickly decreases.

2. The shapes of the graphs for concentration of ASC proteins in the case $k_{1}=1, k_{1}=0.66$, and $k_{1}=0.645$ does not change considerably. However, for $k_{1}=0.6$ the function $x_{1}(t)$ tends to zero. This corresponds to inhibition of mechanoreceptor's formation.
3. For the values of the coefficient $k_{1}$ listed above, variations of the graphs of concentrations $x_{i}(t), i \neq 2$, of other components of CRC have similar characters.

4. The graph of the function $x_{2}(t)$, i.e., content of HAIRY, in general repeats the corresponding curve for $k_{2}=1$, with slightly higher numerical values. This result is biologically reasonable, since $A S$ - $C$ mutants have reduced levels of the SCRT protein which suppresses of hairy expression.

\section{Conclusions}

The main goal of this investigation was to create an improved model of CRC functioning. The simulation results are in good agreement with known literature data. So, the model developed reflects the pattern of CRC functioning correctly and allows to simulate not only the changes in the ASC content in the case of mutational changes in the corresponding gene, but also evaluate the contribution of these changes in content of other CRC proteins.

\section{REFERENCES}

1] E.C. Lai, V. Orgogozo, "A hidden program in Drosophila periphera neurogenesis revealed: fundamental principles underlying sensory organ diversity," Dev. Biol., vol. 269, pp. 1-17, 2004.

[2] T. Bukharina, D. Furman, V. Golubyatnikov, "A model study of the morphogenesis of D.melanogaster mechanorecetpors," JBCB, vol. 13, pp. 1540006-1-1540006-15, 2015.

[3] G.V. Demidenko, "Systems of differential equations of higher dimensions and delay equations," Siberian mathematical journal, vol, 53:6, pp. $1021-1028,2012$

[4] G.V. Demidenko, N.A. Kolchanov., V.A. Likhoshvai., Yu.G.Matushkin, S.I. Fadeev, "Mathematical simulation of regulatory circuits of gene networks," Comput. Math. Math. Phys., vol. 44, pp. 2166 -2183, 2014.

[5] P.J. Chang, Y.L. Hsiao, A.C. Tien., Y.C, Li, H.Pi, "Negative-feedback regulation of proneural proteins controls the timing of neural precursor division," Development, vol. 135, plp. 3021-3030., 2008.

[6] H. Pi, S.K. Huang, C.Y. Tang, Y.H. Sun, C.T. Chien, "Phyllopod is a target gene of proneural proteins in Drosophila external sensory organ development," Proc Natl Acad Sci USA, vol.101, pp. 8378-8383, 2004. 